\title{
The electronic structure of the sulfonyl and phosphonyl groups: A computational and crystallographic study
}

\author{
Emma Denehy, Jonathan M. White, and Spencer J. Williams* \\ School of Chemistry and Bio21 Molecular Science and Biotechnology Institute, University of \\ Melbourne, Parkville, Victoria, Australia 3010. \\ Email: sjwill@unimelb.edu.au
}

\section{TABLE OF CONTENTS}

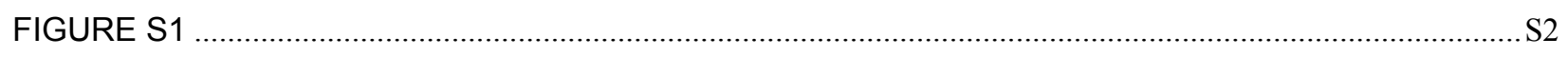

SYNTHESIS OF METHANESULFONIC ACID ESTERS (MESYLATES) ....................................................S3

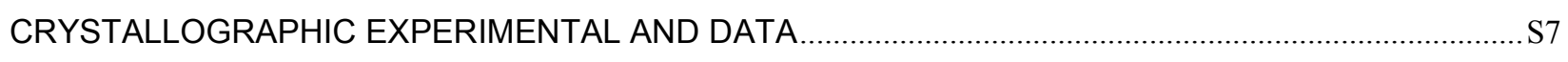

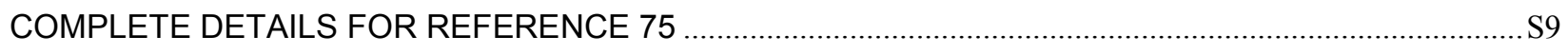

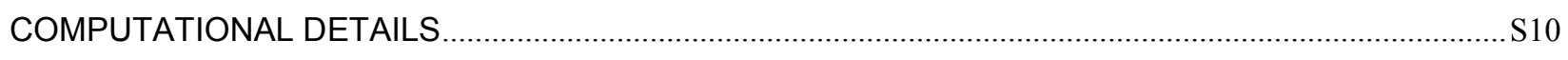

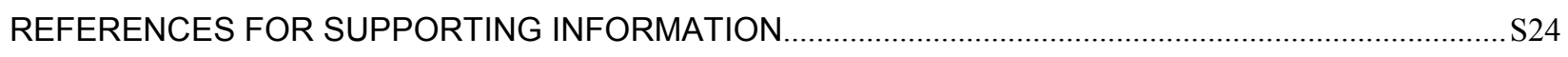


Figure $\mathbf{S 1}$

Structure-reactivity correlation plot of $\mathrm{p} K_{\mathrm{a}}(\mathrm{ROH})$ vs. S-O $\mathrm{O}_{\mathrm{nb}}$ bond length. Sulfate monoesters $(\bullet)$, sulfamates ( $\mathbf{\square})$ and mesylates (०).

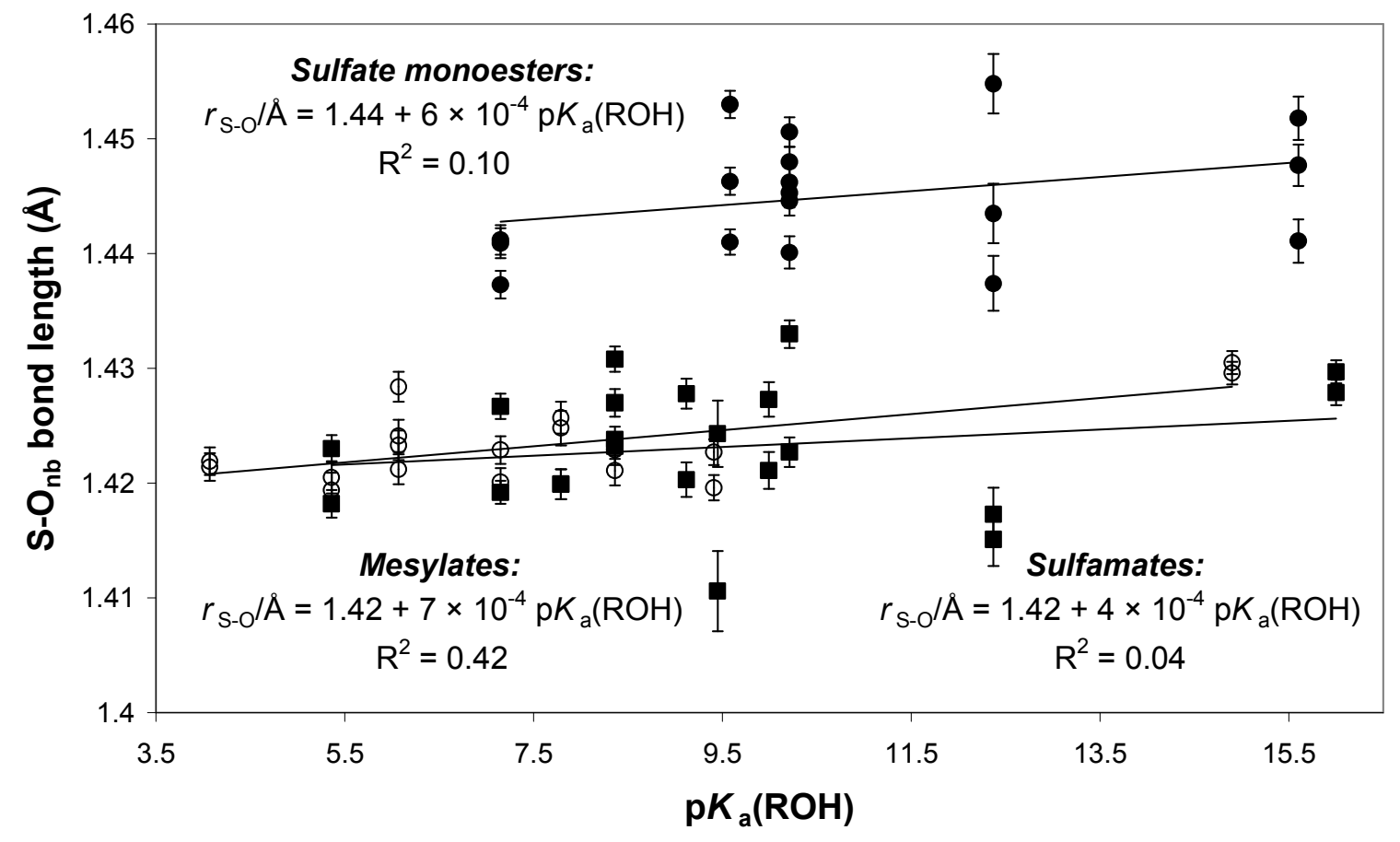




\section{Synthesis of Methanesulfonic Acid Esters (Mesylates)}

\section{General Experimental}

All solvents used for reactions were deoxygenated and dried prior to use following the method of Pangborn and coworkers, ${ }^{1}$ using a commercially available system (Glass Contour Solvent Systems). All melting points were obtained using an Electrothermal melting point apparatus and are uncorrected. ${ }^{1} \mathrm{H}$ and ${ }^{13} \mathrm{C}$ NMR spectra were obtained on a Varian UnityPlus400 instrument (399.7 MHz for ${ }^{1} \mathrm{H}, 100.5 \mathrm{MHz}$ for ${ }^{13} \mathrm{C}$ ). Internal standards employed for NMR spectra were (residual) chloroform $\left({ }^{1} \mathrm{H}, \delta 7.24 ;{ }^{13} \mathrm{C}, \delta 77.0 \mathrm{ppm}\right)$ or internal tetramethylsilane $\left({ }^{1} \mathrm{H},{ }^{13} \mathrm{C}, \delta 0.00\right.$ $\mathrm{ppm})$ for samples run in deuteriochloroform $\left(\mathrm{CDCl}_{3}\right)$.

\section{General procedure for the synthesis of aryl mesylates}

Unless otherwise specified, the procedure of Yamada and coworkers ${ }^{2}$ was employed in the synthesis of aryl mesylates. Triethylamine (1.05 equiv), then methanesulfonyl chloride (1.10 equiv) were added to a solution of the parent phenol (1.0 equiv.) in dry dicholoromethane under $\mathrm{N}_{2}$ at $0{ }^{\circ} \mathrm{C}$. The reaction was then allowed to stir at room temperature $(1-3 \mathrm{~h})$, then was quenched by addition of water. The organic phase was washed with aqueous $\mathrm{HCl}(1 \mathrm{M}, 50 \mathrm{~mL})$, saturated aqueous sodium bicarbonate solution $(50 \mathrm{~mL})$ and water $(50 \mathrm{~mL})$, then dried over $\mathrm{MgSO}_{4}$. The solvent was removed under reduced pressure to yield the crude mesylate, which was recrystallised (ethyl acetate/petroleum spirits b.r. $40-60^{\circ} \mathrm{C}$ ) to yield the pure mesylate.

2,4-Dinitrophenyl mesylate (1): 2,4-Dinitrophenol (2.59 g, $14.1 \mathrm{mmol})$, triethylamine (2.05 mL, $14.8 \mathrm{mmol})$ and methanesulfonyl chloride $(1.20 \mathrm{~mL}, 15.5 \mathrm{mmol})$ according to the general procedure yielded 2,4-dinitrophenyl mesylate as fine yellow needles $(2.99 \mathrm{~g}, 11.4 \mathrm{mmol}, 81 \%)$, m.p. $84-86{ }^{\circ} \mathrm{C}\left(\right.$ lit. $\left.^{3} 83-84{ }^{\circ} \mathrm{C}\right) .{ }^{1} \mathrm{H}$ NMR $\left(400 \mathrm{MHz}, \mathrm{CDCl}_{3}\right) \delta 3.45(3 \mathrm{H}, \mathrm{s}, \mathrm{Me}), 7.80(1 \mathrm{H}, \mathrm{d}, J 9.2$ 
$\mathrm{Hz}, \mathrm{Ar}), 8.55(1 \mathrm{H}, \mathrm{dd}, J$ 9.2, $2.8 \mathrm{~Hz}, \mathrm{Ar}), 8.91(1 \mathrm{H}, \mathrm{d}, J 2.8 \mathrm{~Hz}, \mathrm{Ar}) .{ }^{13} \mathrm{C} \mathrm{NMR}\left(100 \mathrm{MHz}, \mathrm{CDCl}_{3}\right)$ $\delta 39.6(\mathrm{Me}), 122.0,126.5,129.1,141.9,145.4,145.5(\mathrm{Ar})$.

3,4-Dinitrophenyl mesylate (2): 3,4-Dinitrophenol (0.84 g $4.6 \mathrm{mmol})$, triethylamine (0.66 mL, $4.8 \mathrm{mmol})$ and methanesulfonyl chloride $(0.39 \mathrm{~mL}, 5.0 \mathrm{mmol})$ according to the general procedure yielded 3,4-dinitrophenyl mesylate as pale yellow plates (1.01 g, 3.85 mmol, 85\%), m.p. 99-100 ${ }^{\circ} \mathrm{C} .{ }^{1} \mathrm{H}$ NMR (400 MHz, $\left.\mathrm{CDCl}_{3}\right) \delta 3.34(3 \mathrm{H}, \mathrm{s}, \mathrm{Me}), 7.70(1 \mathrm{H}, \mathrm{dd}, J 9.0,2.8 \mathrm{~Hz}, \mathrm{Ar}), 7.83(1 \mathrm{H}, \mathrm{d}$, $J 2.4 \mathrm{~Hz}, \mathrm{Ar}) 8.05(1 \mathrm{H}, \mathrm{d}, J 8.8 \mathrm{~Hz}, \mathrm{Ar}) .{ }^{13} \mathrm{C} \mathrm{NMR}\left(100 \mathrm{MHz}, \mathrm{CDCl}_{3}\right) \delta 38.8(\mathrm{Me}), 119.0,126.6$, 127.1, 140.7, 143.9, $151.2(\mathrm{Ar})$.

4-Nitro-3-trifluoromethylphenyl mesylate (3): 4-Nitro-3-trifluoromethylphenol (0.95 g, 4.6 $\mathrm{mmol})$, triethylamine $(0.70 \mathrm{~mL}, 5.0 \mathrm{mmol})$ and methanesulfonyl chloride $(0.41 \mathrm{~mL}, 5.3 \mathrm{mmol})$ according to the general procedure yielded 4-nitro-3-trifluoromethylphenyl mesylate as colourless needles (0.99 g, $3.5 \mathrm{mmol}, 75 \%)$, m.p. 58.5-60.5 ${ }^{\circ} \mathrm{C} .{ }^{1} \mathrm{H}$ NMR $\left(400 \mathrm{MHz}, \mathrm{CDCl}_{3}\right) \delta$ $3.31(3 \mathrm{H}, \mathrm{s}, \mathrm{Me}), 7.68(1 \mathrm{H}, \mathrm{dd}, J$ 9.0, $2.4 \mathrm{~Hz}, \mathrm{Ar}) 7.70(1 \mathrm{H}, \mathrm{d}, J 2.4 \mathrm{~Hz}, \mathrm{Ar}), 8.02(1 \mathrm{H}, \mathrm{d}, J 9.2$ $\mathrm{Hz}, \mathrm{Ar}) .{ }^{13} \mathrm{C} \mathrm{NMR}\left(100 \mathrm{MHz}, \mathrm{CDCl}_{3}\right) \delta 38.4(\mathrm{Me}), 121.3$ (q, J $\left.274 \mathrm{~Hz}, \mathrm{CF}_{3}\right), 122.3$ (q, J 5.3 Hz, Ar), 125.9 (q, J 35 Hz, Ar), 126.8, 127.7, 146.2, 151.3 (Ar).

4-Nitrophenyl mesylate (4): 4-Nitrophenol (3.00 g, $21.6 \mathrm{mmol})$, triethylamine (3.14 mL, 22.7 mmol) and methanesulfonyl chloride $(1.84 \mathrm{~mL}, 23.8 \mathrm{mmol})$ according to the general procedure yielded 4-nitrophenyl mesylate as a pale yellow, finely divided solid (4.25 g, $19.6 \mathrm{mmol}, 91 \%$ ),

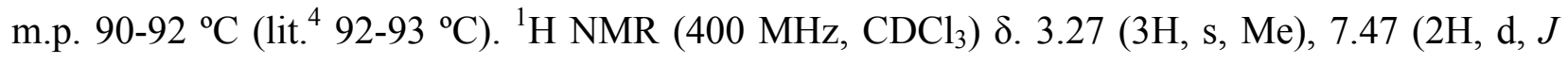


$9.4 \mathrm{~Hz}, \mathrm{Ar}), 8.32(2 \mathrm{H}, \mathrm{d}, J 9.0 \mathrm{~Hz}, \mathrm{Ar}) .{ }^{13} \mathrm{C} \mathrm{NMR}\left(100 \mathrm{MHz}, \mathrm{CDCl}_{3}\right) \delta 38.3(\mathrm{Me}), 122.8,125.8$, $146.3,153.2(\mathrm{Ar})$.

4-Cyanophenyl mesylate (5): 4-Cyanophenol (3.00 g, $25.2 \mathrm{mmol}$ ), triethylamine (3.67 mL, 26.5 $\mathrm{mmol})$ and methanesulfonyl chloride $(2.16 \mathrm{~mL}, 27.9 \mathrm{mmol})$ according to the general procedure yielded 4-cyanophenyl mesylate ester as colourless cubic crystals $(4.96 \mathrm{~g}, 100 \%)$, m.p. $94-97{ }^{\circ} \mathrm{C}$ (lit. $\left.{ }^{5} 89-90{ }^{\circ} \mathrm{C}\right) .{ }^{1} \mathrm{H}$ NMR $\left(400 \mathrm{MHz}, \mathrm{CDCl}_{3}\right) \delta 3.24(3 \mathrm{H}, \mathrm{s}, \mathrm{Me}), 7.43(2 \mathrm{H}, \mathrm{d}, J 8.8 \mathrm{~Hz}, \mathrm{Ar}), 7.75$ $(2 \mathrm{H}, \mathrm{d}, J 8.8 \mathrm{~Hz}, \mathrm{Ar}) .{ }^{13} \mathrm{C} \mathrm{NMR}\left(100 \mathrm{MHz}, \mathrm{CDCl}_{3}\right) \delta 38.1(\mathrm{Me}), 111.3(\mathrm{Ar}), 117.7(\mathrm{C} \equiv \mathrm{N})$, $123.1,134.3,152.0(\mathrm{Ar})$.

3-Nitrophenyl mesylate (6): 3-Nitrophenol (3.00 g, $21.6 \mathrm{mmol})$, triethylamine (3.14 mL, 22.7 mmol) and methanesulfonyl chloride $(1.84 \mathrm{~mL}, 23.8 \mathrm{mmol})$ according to the general procedure yielded 3-nitrophenyl mesylate ester as large pale yellow cubic crystals $(4.41 \mathrm{~g}, 20.3 \mathrm{mmol}$, 94\%), m.p. 66-69 ${ }^{\circ} \mathrm{C}$ (lit. $\left.{ }^{6} 60{ }^{\circ} \mathrm{C}\right) .{ }^{1} \mathrm{H}$ NMR (400 MHz, $\left.\mathrm{CDCl}_{3}\right) \delta 3.28(3 \mathrm{H}, \mathrm{s}, \mathrm{Me}), 7.66(2 \mathrm{H}, \mathrm{m}$, Ar), $8.15(1 \mathrm{H}, \mathrm{s}, \mathrm{Ar}), 8.22(1 \mathrm{H}, \mathrm{d}, J 7.2 \mathrm{~Hz}, \mathrm{Ar}) .{ }^{13} \mathrm{C} \mathrm{NMR}\left(100 \mathrm{MHz}, \mathrm{CDCl}_{3}\right) \delta 38.1(\mathrm{Me})$, $117.2,122.3,128.6,130.9,148.9,149.0(\mathrm{Ar})$.

4-Chlorophenyl mesylate (7): 4-Chlorophenol (3.00 g, $23.3 \mathrm{mmol})$, triethylamine (3.40 $\mathrm{mL}$, $24.6 \mathrm{mmol})$ and methanesulfonyl chloride $(2.00 \mathrm{~mL}, 25.8 \mathrm{mmol})$ according to the general procedure yielded 4-chlorophenyl mesylate ester as colourless cubic crystals $(4.17 \mathrm{~g}, 20.2 \mathrm{mmol}$, 87\%), m.p. $70-71{ }^{\circ} \mathrm{C}$ (lit. $\left.{ }^{7} 68-69^{\circ} \mathrm{C}\right) .{ }^{1} \mathrm{H}$ NMR $\left(400 \mathrm{MHz}, \mathrm{CDCl}_{3}\right) \delta 3.15(3 \mathrm{H}, \mathrm{s}, \mathrm{Me}), 7.23(2 \mathrm{H}$, d, $J 8.8 \mathrm{~Hz}, \mathrm{Ar}), 7.38(2 \mathrm{H}, \mathrm{d}, J 8.8 \mathrm{~Hz}, \mathrm{Ar}) .{ }^{13} \mathrm{C} \mathrm{NMR}\left(100 \mathrm{MHz}, \mathrm{CDCl}_{3}\right) \delta 37.5(\mathrm{Me}), 123.5$, $130.1,133.1,147.5(\mathrm{Ar})$ 
4-Nitrobenzyl mesylate (8): The procedure of Allen and coworkers ${ }^{8}$ was used in the synthesis of 4-nitrobenzyl mesylate. Triethylamine $(3.66 \mathrm{~mL}, 26.2 \mathrm{mmol})$ was added to a solution of 4nitrobenzyl alcohol $(2.00 \mathrm{~g}, 13.1 \mathrm{mmol})$ in dry THF $(20 \mathrm{~mL})$ under $\mathrm{N}_{2}$. The reaction was cooled to $-15{ }^{\circ} \mathrm{C}$ and methanesulfonyl chloride $(1.22 \mathrm{~mL}, 15.7 \mathrm{mmol})$ was added dropwise, resulting in formation of a pale yellow precipitate. The mixture was allowed to stir at $-15^{\circ} \mathrm{C}$ for 30 minutes followed by addition of aqueous $\mathrm{HCl}(1 \mathrm{M}, 50 \mathrm{~mL})$, then water $(20 \mathrm{~mL})$. The aqueous phase was extracted with ethyl acetate $(50 \mathrm{~mL} \times 2)$. The combined organic phases were dried $\left(\mathrm{MgSO}_{4}\right)$ and the solvent removed under reduced pressure at room temperature. The crude iridescent colourless residue was redissolved in ethyl acetate $(100 \mathrm{~mL})$, then petroleum spirits (b.r. $\left.40-60{ }^{\circ} \mathrm{C}\right)$ was added until the product precipitated as colourless, finely divided iridescent plates ( $2.68 \mathrm{~g}, 11.6$ mmol, 89\%), m.p. $110-111^{\circ} \mathrm{C}$ (lit. $\left.{ }^{9} 102-104{ }^{\circ} \mathrm{C}\right) .{ }^{1} \mathrm{H}$ NMR (400 MHz, $\left.\mathrm{CDCl}_{3}\right) \delta 3.07$ (3H, s, Me), $5.34\left(2 \mathrm{H}, \mathrm{s}, \mathrm{CH}_{2}\right), 7.60(2 \mathrm{H}, \mathrm{d}, J 8.4 \mathrm{~Hz}, \mathrm{Ar}), 8.27(2 \mathrm{H}, \mathrm{d}, J 8.4 \mathrm{~Hz}, \mathrm{Ar}) .{ }^{13} \mathrm{C} \mathrm{NMR}(100 \mathrm{MHz}$, $\left.\mathrm{CDCl}_{3}\right) 38.2(\mathrm{Me}), 69.0\left(\mathrm{CH}_{2}\right), 124.1,128.9,140.6,148.3(\mathrm{Ar})$. 


\section{Crystallographic Experimental and Data}

Crystallisation conditions for mesylates and $p K_{a}$ values for the parent alcohols.

2,4-dinitrophenyl (1)
3,4-dinitrophenyl (2)
4-nitro-3-
trifluoromethylphenyl

(3)

4-nitrophenyl (4)

4-cyanophenyl (5)

3-nitrophenyl (6)

4-chlorophenyl (7)

4-nitrobenzyl (8)

\section{Crystallisation conditions}

EtOAc, slow evaporation at $4^{\circ} \mathrm{C}$

EtOAc/pet. Spirit (b.r. 40-

$60{ }^{\circ} \mathrm{C}$ ), vapour diffusion, room temp.

EtOAc/pet. Spirit (b.r. 40-

$60{ }^{\circ} \mathrm{C}$ ), vapour diffusion, room temp.

Ethyl acetate, slow evaporation, room temp.

Toluene, slow evaporation at $4{ }^{\circ} \mathrm{C}$

EtOAc/pet. Spirit (b.r. 40$60{ }^{\circ} \mathrm{C}$ ), slow cooling, room temp

\section{EtOAc/pet. Spirit (b.r. 40-} $60{ }^{\circ} \mathrm{C}$ ), slow cooling, room temp

$\mathrm{CH}_{2} \mathrm{Cl}_{2} / \mathrm{Et}_{2} \mathrm{O}$, slow evaporation at room temperature
Crystal appearance $\mid \mathrm{p} K_{\mathrm{a}}(\mathrm{ROH})$ colourless blocks $\quad \mathrm{p} K_{\mathrm{a}}=$

$4.07^{10}$

$\mathrm{p} K_{\mathrm{a}}=$

$5.36^{10}$

colourless blocks $\quad \mathrm{p} K_{\mathrm{a}}=$

$6.07^{10}$

colourless blocks $\quad \mathrm{p} K_{\mathrm{a}}=$

$7.15^{10}$

$\mathrm{p} K_{\mathrm{a}}=$

$7.79^{10}$

$\mathrm{p} K_{\mathrm{a}}=$

$8.36^{10}$

$\mathrm{p} K_{\mathrm{a}}=$

$9.41^{10}$

colourless blocks $\quad \mathrm{p} K_{\mathrm{a}}=$

$14.9^{11}$ 


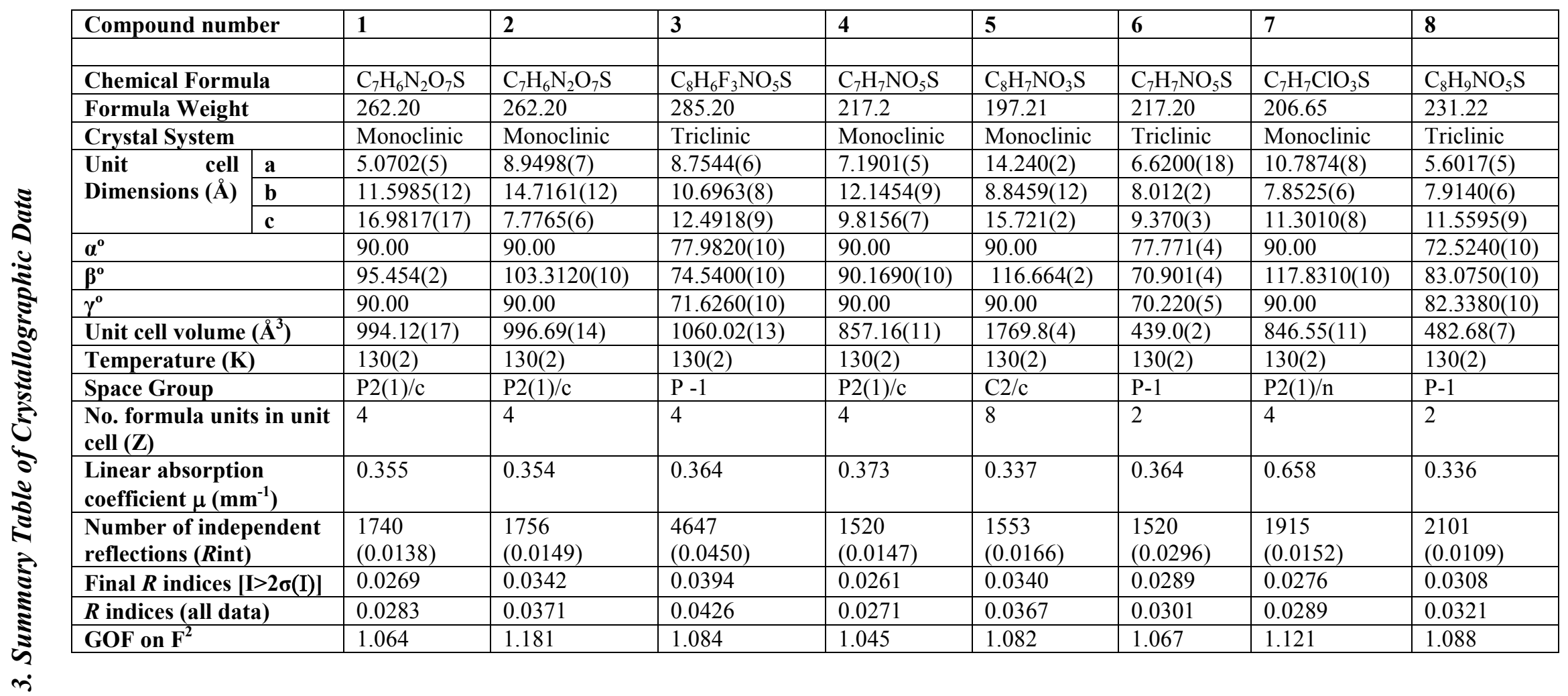




\section{Complete Details for Reference 75}

Gaussian 03, Revision B.04, M. J. Frisch, G. W. Trucks, H. B. Schlegel, G. E. Scuseria, M. A. Robb, J. R. Cheeseman, J. A. Montgomery, Jr., T. Vreven, K. N. Kudin, J. C. Burant, J. M. Millam, S. S. Iyengar, J. Tomasi, V. Barone, B. Mennucci, M. Cossi, G. Scalmani, N. Rega, G. A. Petersson, H. Nakatsuji, M. Hada, M. Ehara, K. Toyota, R. Fukuda, J. Hasegawa, M. Ishida, T. Nakajima, Y. Honda, O. Kitao, H. Nakai, M. Klene, X. Li, J. E. Knox, H. P. Hratchian, J. B. Cross, C. Adamo, J. Jaramillo, R. Gomperts, R. E. Stratmann, O. Yazyev, A. J. Austin, R. Cammi, C. Pomelli, J. W. Ochterski, P. Y. Ayala, K. Morokuma, G. A. Voth, P. Salvador, J. J. Dannenberg, V. G. Zakrzewski, S. Dapprich, A. D. Daniels, M. C. Strain, O. Farkas, D. K. Malick, A. D. Rabuck, K. Raghavachari, J. B. Foresman, J. V. Ortiz, Q. Cui, A. G. Baboul, S. Clifford, J. Cioslowski, B. B. Stefanov, G. Liu, A. Liashenko, P. Piskorz, I. Komaromi, R. L. Martin, D. J. Fox, T. Keith, M. A. Al-Laham, C. Y. Peng, A. Nanayakkara, M. Challacombe, P. M. W. Gill, B. Johnson, W. Chen, M. W. Wong, C. Gonzalez, and J. A. Pople, Gaussian, Inc., Pittsburgh PA, 2003. 


\section{Computational Details}

All geometries are specified below in Cartesian coordinates in the standard orientation.

Sulfur trioxide $\left(\mathrm{SO}_{3}\right)$ at $B 3 L Y P / 6311++G(3 d f, 2 p)$

Energy: -623.936314647 Hartrees

Cartesian Coordinates (Standard Orientation)

$\begin{array}{lrrr}16 & 0.000000 & 0.000000 & 0.000000 \\ 8 & 0.000000 & 1.425308 & 0.000000 \\ 8 & 1.234353 & -0.712654 & 0.000000 \\ 8 & -1.234353 & -0.712654 & 0.000000\end{array}$

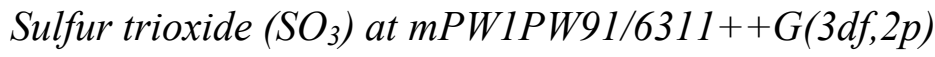

Energy: -623.879497462 Hartrees

Cartesian Coordinates (Standard Orientation)

$\begin{array}{lrrr}16 & 0.000000 & 0.000000 & 0.000000 \\ 8 & 0.000000 & 1.417008 & 0.000000 \\ 8 & 1.227165 & -0.708504 & 0.000000 \\ 8 & -1.227165 & -0.708504 & 0.000000\end{array}$

Methyl phosphate dianion $\left(\mathrm{MeOPO}_{3}{ }^{2-}\right)$ at $\mathrm{mPW1PW91/6311++G}$

Energy: -682.005805017 Hartrees

Cartesian Coordinates (Standard Orientation)

$\begin{array}{lrrr}6 & -2.192585 & 0.056974 & -0.000243 \\ 8 & -1.040167 & 0.890700 & -0.002615 \\ 15 & 0.570567 & -0.066369 & 0.000196 \\ 8 & 1.656792 & 1.134743 & -0.002605 \\ 8 & 0.449775 & -0.945356 & -1.368963 \\ 8 & 0.448956 & -0.938237 & 1.373850 \\ 1 & -2.219635 & -0.591308 & -0.887800 \\ 1 & -2.218081 & -0.588175 & 0.889613 \\ 1 & -3.088131 & 0.698358 & -0.000631\end{array}$

Methyl phosphate dianion $\left(\mathrm{MeOPO}_{3}{ }^{2-}\right)$ at $\mathrm{mPW} 1 \mathrm{PW} 91 / 6311++G(3 d f, 2 p)$

Energy: -682.284202633 Hartrees

\begin{tabular}{|c|c|c|c|}
\hline \multicolumn{4}{|c|}{ Cartesian Coordinates (Standard Orientation) } \\
\hline 6 & -2.096149 & 0.044505 & -0.000091 \\
\hline 8 & -0.964481 & 0.827690 & -0.001270 \\
\hline 15 & 0.536501 & -0.059255 & 0.000079 \\
\hline 8 & 1.539416 & 1.072677 & -0.000932 \\
\hline 8 & 0.451451 & -0.879671 & -1.277934 \\
\hline . & 0.450802 & -0.876766 & 1.279946 \\
\hline
\end{tabular}




$\begin{array}{rrrr}1 & -2.149865 & -0.608416 & -0.888239 \\ 1 & -2.144465 & -0.613011 & 0.884882 \\ 1 & -2.993793 & 0.691777 & 0.004249\end{array}$

Methyl sulfate anion $\left(\mathrm{MeOSO}_{3}^{-}\right)$at $\mathrm{mPW} 1 \mathrm{PW} 91 / 6311++G$

\section{Energy: -738.765712504 Hartrees}

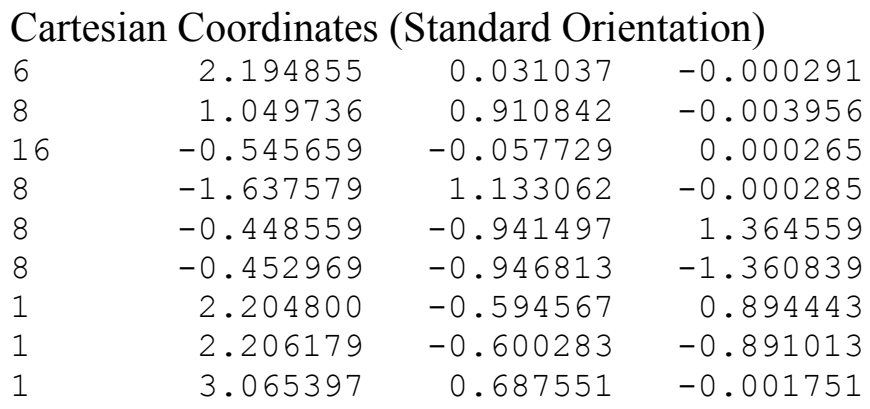

Methyl sulfate anion $\left(\mathrm{MeOSO}_{3}^{-}\right)$at mPW1PW91/6311++G(3df,2p)

\section{Energy: -739.148180154 Hartrees}

Cartesian Coordinates (Standard Orientation)
$\begin{array}{lrrr}6 & 2.070136 & 0.020887 & 0.000164 \\ 8 & 0.909674 & 0.810741 & 0.003776 \\ 16 & -0.499845 & -0.048755 & -0.000295 \\ 8 & -1.472466 & 1.013469 & -0.012543 \\ 8 & -0.449220 & -0.831109 & 1.218397 \\ 8 & -0.434423 & -0.845839 & -1.208836 \\ 1 & 2.114389 & -0.617607 & 0.887116 \\ 1 & 2.112817 & -0.610046 & -0.892164 \\ 1 & 2.920969 & 0.704324 & 0.002429\end{array}$

Methyl hydrogen phosphate $\left(\mathrm{MeOPO}_{2} \mathrm{OH}\right)$ at $m P W 1 P W 91 / 6311++G(3 d f, 2 p)$

Energy: -683.022504569 Hartrees

Cartesian Coordinates (Standard Orientation)
$\begin{array}{lrrr}6 & 2.154060 & -0.102782 & -0.034179 \\ 8 & 0.939152 & -0.514796 & -0.592923 \\ 15 & -0.413202 & 0.124563 & 0.105147 \\ 8 & -1.503109 & -0.569078 & -0.894179 \\ 8 & -0.409839 & 1.586126 & -0.120250 \\ 8 & -0.577010 & -0.488008 & 1.451134 \\ 1 & 2.294177 & 0.978017 & -0.141503 \\ 1 & 2.210497 & -0.354788 & 1.030659 \\ 1 & 2.960983 & -0.618961 & -0.560345 \\ 1 & -1.785545 & -1.369963 & -0.451196\end{array}$

Methyl hydrogen sulfate $\left(\mathrm{MeOSO}_{2} \mathrm{OH}\right)$ at mPW1PW91/6311++G(3df,2p)

Energy: -739.658450184 Hartrees 


Cartesian Coordinates (Standard Orientation)
$\begin{array}{lrrr}6 & 2.142345 & -0.079314 & -0.021934 \\ 8 & 0.886727 & -0.629335 & -0.449906 \\ 16 & -0.392538 & 0.128078 & 0.069353 \\ 8 & -1.467270 & -0.757616 & -0.651002 \\ 8 & -0.453783 & 1.416157 & -0.510935 \\ 8 & -0.499107 & -0.049673 & 1.476177 \\ 1 & 2.246110 & 0.940592 & -0.387064 \\ 1 & 2.213708 & -0.108696 & 1.063784 \\ 1 & 2.900099 & -0.715785 & -0.465901 \\ 1 & -1.665920 & -1.525739 & -0.103534\end{array}$

Methyl phosphamate anion $\left(\mathrm{MeOPO}_{2} \mathrm{NH}_{2}^{-}\right)$at mPW1PW91/6311++G(3df,2p)

Energy: -663.134523824 Hartrees

Cartesian Coordinates (Standard Orientation)
$\begin{array}{lrrr}6 & 2.159245 & 0.163097 & -0.000325 \\ 8 & 0.918854 & 0.808345 & -0.001980 \\ 15 & -0.411874 & -0.197844 & 0.000278 \\ 7 & -1.634833 & 0.978960 & -0.000750 \\ 8 & -0.436381 & -0.900376 & 1.307514 \\ 8 & -0.437965 & -0.904282 & -1.304811 \\ 1 & 2.280733 & -0.466074 & 0.888740 \\ 1 & 2.281630 & -0.469015 & -0.887191 \\ 1 & 2.943037 & 0.925938 & -0.001211 \\ 1 & -1.597897 & 1.556895 & -0.827762 \\ 1 & -1.597088 & 1.559132 & 0.824658\end{array}$

\section{Methyl sulfamate $\left(\mathrm{MeOSO}_{2} \mathrm{NH}_{2}\right)$ at mPW1PW91/6311++G(3df,2p)}

\section{Energy: -719.788430403 Hartrees}

\begin{tabular}{lrrr}
\multicolumn{4}{l}{ Cartesian Coordinates (Standard Orientation) } \\
6 & -2.150559 & 0.147704 & -0.000612 \\
8 & -0.875002 & 0.801179 & -0.005121 \\
16 & 0.387964 & -0.168104 & 0.000565 \\
7 & 1.597214 & 0.903753 & -0.001592 \\
8 & 0.414804 & -0.870146 & -1.234909 \\
8 & 0.410822 & -0.860729 & 1.241461 \\
1 & -2.259727 & -0.468823 & -0.891257 \\
1 & -2.258861 & -0.458105 & 0.897430 \\
1 & -2.886943 & 0.944793 & -0.005095 \\
1 & 1.656813 & 1.451339 & 0.843471 \\
1 & 1.659163 & 1.445528 & -0.850227
\end{tabular}

Methyl phosphamate dianion $\left(\mathrm{MeOPO}_{2} \mathrm{NH}^{2-}\right)$ at mPW1PW91/6311++G(3df,2p)

\section{Energy: -662.363871578 Hartrees}

Cartesian Coordinates (Standard Orientation)

6

$$
\begin{array}{lll}
2.116016 & -0.104331 & -0.065142
\end{array}
$$




$\begin{array}{lrrr}8 & 0.951624 & -0.584652 & -0.626061 \\ 15 & -0.519113 & 0.086123 & 0.055778 \\ 7 & -1.686154 & -0.632978 & -0.801059 \\ 8 & -0.374587 & 1.563757 & -0.236175 \\ 8 & -0.405476 & -0.328606 & 1.516684 \\ 1 & 2.210289 & 0.988581 & -0.180485 \\ 1 & 2.176363 & -0.321584 & 1.014799 \\ 1 & 2.986027 & -0.576564 & -0.559514 \\ 1 & -1.851487 & -1.529426 & -0.348782\end{array}$

Methyl sulfamate ester anion $\left(\mathrm{MeOSO}_{2} \mathrm{NH}\right)$ at mPW1PW91/6311++G(3df,2p)

\section{Energy: -719.230278515 Hartrees}

Cartesian Coordinates (Standard Orientation)
6
$\begin{array}{lrrr}6 & 2.092307 & -0.081851 & -0.047866 \\ 8 & 0.903556 & -0.624284 & -0.559609 \\ 16 & -0.492569 & 0.072370 & 0.042418 \\ 7 & -1.618272 & -0.658839 & -0.689179 \\ 8 & -0.382423 & 1.455418 & -0.363724 \\ 8 & -0.373591 & -0.165602 & 1.473619 \\ 1 & 2.172069 & 0.984004 & -0.281652 \\ 1 & 2.150813 & -0.209707 & 1.037279 \\ 1 & 2.919934 & -0.616185 & -0.519730 \\ 1 & -1.767983 & -1.537296 & -0.205420\end{array}$

Methyl methylphosphonate anion $\left(\mathrm{MeOPO}_{2} \mathrm{CH}_{3}^{-}\right)$at mPW1PW91/6311++G(3df,2p)

\section{Energy: -647.071480399 Hartrees}

$\begin{array}{lrrr}\text { Cartesian Coordinates (Standard Orientation) } \\ 6 & -2.175613 & 0.233442 & -0.000708 \\ 8 & -0.910142 & 0.828581 & -0.003216 \\ 15 & 0.380543 & -0.220547 & 0.000350 \\ 6 & 1.680110 & 1.054887 & -0.000939 \\ 8 & 0.392988 & -0.952993 & -1.296330 \\ 8 & 0.389780 & -0.947022 & 1.300434 \\ 1 & -2.926713 & 1.027444 & 0.028935 \\ 1 & -2.311406 & -0.412999 & 0.873445 \\ 1 & -2.335356 & -0.369892 & -0.901344 \\ 1 & 2.646433 & 0.550891 & 0.001718 \\ 1 & 1.604181 & 1.679374 & 0.888741 \\ 1 & 1.606731 & 1.674887 & -0.893965\end{array}$

Methyl methanesulfonate $\left(\mathrm{MeOSO}_{2} \mathrm{CH}_{3}\right)$ at $\mathrm{mPW1PW91/6311++G(3df,2p)}$

Energy: -703.739693108 Hartrees

\section{Cartesian Coordinates (Standard Orientation)}

$\begin{array}{lrrr}6 & 2.161323 & 0.211553 & -0.000115 \\ 8 & 0.862468 & 0.818233 & -0.000863 \\ 16 & -0.362395 & -0.193561 & 0.000052 \\ 6 & -1.645279 & 1.006128 & 0.000082\end{array}$




$\begin{array}{rrrr}8 & -0.371109 & -0.916411 & 1.231223 \\ 8 & -0.372262 & -0.917435 & -1.230502 \\ 1 & 2.866889 & 1.035743 & 0.000868 \\ 1 & 2.293267 & -0.394687 & -0.894500 \\ 1 & 2.291889 & -0.395590 & 0.893882 \\ 1 & -2.578932 & 0.449254 & 0.001047 \\ 1 & -1.562474 & 1.609912 & -0.898264 \\ 1 & -1.561371 & 1.611161 & 0.897483\end{array}$

Methyl methylphosphonate dianion $\left(\mathrm{MeOPO}_{2} \mathrm{CH}_{2}{ }^{2-}\right)$ at mPW1PW91/6311++G(3df,2p)

Energy: -646.278677392 Hartrees

Cartesian Coordinates (Standard Orientation)
6
$\begin{array}{lrrr}6.132361 & 0.215273 & -0.000149 \\ 8 & 0.922082 & 0.882295 & -0.003271 \\ 15 & -0.494004 & -0.160106 & 0.000271 \\ 6 & -1.840307 & 0.942029 & -0.001734 \\ 8 & -0.335298 & -0.921238 & 1.297142 \\ 8 & -0.336383 & -0.928055 & -1.292695 \\ 1 & 2.958350 & 0.950427 & -0.000881 \\ 1 & 2.250649 & -0.431225 & -0.885431 \\ 1 & 2.248241 & -0.426952 & 0.888486 \\ 1 & -1.901802 & 1.551379 & 0.904262 \\ 1 & -1.900917 & 1.550147 & -0.908614\end{array}$

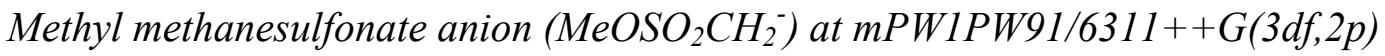

\section{Energy: - 703.146239293 Hartrees}

\begin{tabular}{lrrr}
\multicolumn{4}{l}{ Cartesian Coordinates (Standard Orientation) } \\
6 & 2.120379 & -0.012257 & 0.173803 \\
8 & 0.900129 & -0.057485 & 0.863835 \\
16 & -0.477868 & 0.008589 & -0.124059 \\
6 & -1.780684 & -0.059213 & 0.846881 \\
8 & -0.314325 & -1.174892 & -0.946318 \\
8 & -0.316525 & 1.293108 & -0.777776 \\
1 & 2.219250 & -0.857329 & -0.515052 \\
1 & 2.221636 & 0.917353 & -0.395491 \\
1 & 2.919038 & -0.063459 & 0.918789 \\
1 & -1.953398 & 0.832990 & 1.432013 \\
1 & -1.953053 & -1.024010 & 1.302656
\end{tabular}

Bromosulfate anion $\left(\mathrm{SO}_{3} \mathrm{Br}^{-}\right)$at $\mathrm{mPW} 1 \mathrm{PW} 91 / 6311++G(3 d f, 2 \mathrm{p})$

Energy: -3198.33879571 Hartrees
Cartesian Coordinates (Standard Orientation)
$\begin{array}{lrrr}35 & 1.357877 & -0.000107 & 0.000007 \\ 16 & -1.017955 & -0.000038 & -0.000004 \\ 8 & -1.301006 & 1.387830 & -0.248122 \\ 8 & -1.301956 & -0.908536 & -1.077583 \\ 8 & -1.301840 & -0.478751 & 1.325681\end{array}$


Chlorosulfate anion $\left(\mathrm{SO}_{3} \mathrm{Cl}^{-}\right)$at $m P W 1 P W 91 / 6311++G(3 d f, 2 p)$

Energy: -1084.25993377 Hartrees

Cartesian Coordinates (Standard Orientation)

$\begin{array}{lrrr}17 & -1.651639 & -0.000063 & 0.000064 \\ 16 & 0.526859 & 0.000000 & -0.000020 \\ 8 & 0.818470 & 1.309828 & -0.516612 \\ 8 & 0.818846 & -0.207432 & 1.392453 \\ 8 & 0.818698 & -1.102263 & -0.875936\end{array}$

Fluorosulfate anion $\left(\mathrm{SO}_{3} \mathrm{~F}^{\mathrm{*}}\right)$ at $\mathrm{mPW} 1 \mathrm{PW} 91 / 6311++G(3 d f, 2 p)$

Energy: - 723.880081322 Hartrees

Cartesian Coordinates (Standard Orientation)

$\begin{array}{lrrr}9 & 1.480657 & 0.001434 & -0.000658 \\ 16 & -0.151017 & -0.000234 & 0.000066 \\ 8 & -0.456073 & 1.385663 & -0.241437 \\ 8 & -0.454170 & -0.902700 & -1.079160 \\ 8 & -0.453463 & -0.484108 & 1.321207\end{array}$

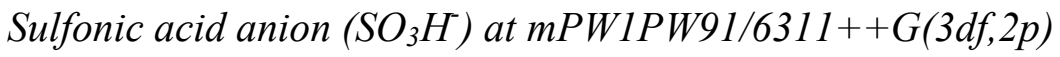

Energy: -624.591750656 Hartrees

Cartesian Coordinates (Standard Orientation)

$\begin{array}{lrrr}1 & 0.000530 & 0.000025 & 1.532246 \\ 16 & 0.000150 & 0.000003 & 0.168886 \\ 8 & -1.385028 & -0.273374 & -0.176333 \\ 8 & 0.455541 & 1.335829 & -0.176470 \\ 8 & 0.929121 & -1.062463 & -0.176499\end{array}$

Hydrogen sulfate anion $\left(\mathrm{SO}_{3} \mathrm{OH}\right)$ at $m P W 1 P W 91 / 6311++G(3 d f, 2 p)$

Energy: -699.846633977 Hartrees

Cartesian Coordinates (Standard Orientation)

$\begin{array}{lrrr}1 & -1.951332 & -0.300259 & -0.000081 \\ 8 & -1.422542 & 0.500433 & -0.000339 \\ 16 & 0.143550 & -0.027264 & 0.000013 \\ 8 & 0.870889 & 1.216726 & -0.000230 \\ 8 & 0.254419 & -0.812918 & -1.212998 \\ 8 & 0.254051 & -0.812180 & 1.213551\end{array}$

Sulfamate anion $\left(\mathrm{SO}_{3} \mathrm{NH}_{2}^{-}\right)$at $m P W 1 P W 91 / 6311++G(3 d f, 2 p)$

Energy: -679.965826107 Hartrees

Cartesian Coordinates (Standard Orientation)

$\begin{array}{llll}7 & -1.534548 & -0.000020 & -0.274273\end{array}$ 


$\begin{array}{lrrr}16 & 0.155212 & 0.000002 & 0.003684 \\ 8 & 0.328444 & -0.000035 & 1.450612 \\ 8 & 0.589263 & -1.226028 & -0.634791 \\ 8 & 0.589215 & 1.226083 & -0.634724 \\ 1 & -1.898477 & 0.815136 & 0.206093 \\ 1 & -1.898459 & -0.815187 & 0.206089\end{array}$

Methanesulfonate anion $\left(\mathrm{SO}_{3} \mathrm{CH}_{3}^{-}\right)$at mPW1PW91/6311++G(3df,2p)

\section{Energy: -663.916407406 Hartrees}

Cartesian Coordinates (Standard Orientation)
$\begin{array}{lrrr}6 & -1.622152 & 0.000054 & 0.000125 \\ 16 & 0.173340 & 0.000042 & -0.000026 \\ 8 & 0.535921 & -1.010529 & -0.984320 \\ 8 & 0.536161 & 1.357668 & -0.382910 \\ 8 & 0.536251 & -0.347304 & 1.367109 \\ 1 & -1.969068 & -0.992927 & 0.278174 \\ 1 & -1.969175 & 0.255974 & -0.998754 \\ 1 & -1.968963 & 0.737276 & 0.721212\end{array}$

Phosphonate dianion $\left(\mathrm{PO}_{3} \mathrm{H}^{2-}\right)$ at $m P W 1 P W 91 / 6311++G(3 d f, 2 p)$

\section{Energy: -567.695919436 Hartrees}

\begin{tabular}{lrrr}
\multicolumn{4}{c}{ Cartesian Coordinates (Standard Orientation) } \\
1 & 0.000391 & 0.000021 & 1.649551 \\
15 & 0.000010 & -0.000012 & 0.171596 \\
8 & 1.449286 & -0.334364 & -0.176021 \\
8 & -1.014269 & -1.087866 & -0.175951 \\
8 & -0.435085 & 1.422250 & -0.175965
\end{tabular}

Hydrogen phosphate dianion $\left(\mathrm{PO}_{3} \mathrm{OH}^{2-}\right)$ at $\mathrm{mPW} 1 \mathrm{PW} 91 / 6311++G(3 d f, 2 \mathrm{p})$

Energy: -642.978832205 Hartrees

\begin{tabular}{llrr}
\multicolumn{4}{c}{ Cartesian Coordinates (Standard Orientation) } \\
1 & 0.000000 & 0.000000 & 0.000000 \\
8 & 0.000000 & 0.000000 & 0.957202 \\
15 & 1.699092 & 0.000000 & 1.307284 \\
8 & 1.716011 & -0.000219 & 2.823023 \\
8 & 2.190214 & 1.277974 & 0.634840 \\
8 & 2.190259 & -1.277759 & 0.634461
\end{tabular}

Phosphamate dianion $\left(\mathrm{PO}_{3} \mathrm{NH}_{2}{ }^{2-}\right)$ at $\mathrm{mPW} 1 \mathrm{PW} 91 / 6311++G(3 d f, 2 p)$

Energy: -623.088243445 Hartrees

Cartesian Coordinates (Standard Orientation)

$\begin{array}{lrrr}7 & -1.570004 & -0.000012 & -0.460319 \\ 15 & 0.179530 & 0.000001 & 0.018794 \\ 8 & 0.133206 & -0.000038 & 1.549171\end{array}$




$\begin{array}{rrrr}8 & 0.695748 & -1.293157 & -0.595185 \\ 8 & 0.695706 & 1.293207 & -0.595122 \\ 1 & -1.950106 & 0.802628 & 0.034711 \\ 1 & -1.950094 & -0.802663 & 0.034703\end{array}$

Methylphosphonate dianion, $\left(\mathrm{PO}_{3} \mathrm{CH}_{3}{ }^{2-}\right)$ at $\mathrm{mPW} 1 \mathrm{PW} 91 / 6311++\mathrm{G}(3 \mathrm{df}, 2 \mathrm{p})$

\section{Energy: -607.026801261 Hartrees}

Cartesian Coordinates (Standard Orientation)
$\begin{array}{lrrr}6 & -1.691204 & 0.000493 & 0.000087 \\ 15 & 0.202618 & -0.000020 & -0.000016 \\ 8 & 0.554404 & 1.394053 & -0.515542 \\ 8 & 0.553864 & -0.250846 & 1.465144 \\ 8 & 0.553301 & -1.143782 & -0.949686 \\ 1 & -2.061521 & 0.173964 & -1.016176 \\ 1 & -2.061289 & 0.794129 & 0.658311 \\ 1 & -2.061789 & -0.966151 & 0.358253\end{array}$

Sulfamoyl bromide $\left(\mathrm{NH}_{2} \mathrm{SO}_{2} \mathrm{Br}\right)$ at mPW1PW91/6311++G(3df,2p)

\section{Energy: -3178.95585247 Hartrees}

\begin{tabular}{lrrr}
\multicolumn{4}{c}{ Cartesian Coordinates (Standard Orientation) } \\
35 & -1.329243 & -0.101280 & 0.000000 \\
16 & 0.908851 & -0.070524 & 0.000000 \\
7 & 1.300511 & 1.501461 & 0.000000 \\
8 & 1.300511 & -0.612605 & 1.250098 \\
8 & 1.300511 & -0.612605 & -1.250098 \\
1 & 1.035071 & 1.982309 & 0.847805 \\
1 & 1.035071 & 1.982309 & -0.847805
\end{tabular}

Sulfamoyl chloride $\left(\mathrm{NH}_{2} \mathrm{SO}_{2} \mathrm{Cl}\right)$ at mPW1PW91/6311++G(3df,2p)

\section{Energy: -1064.87985612 Hartrees}

\section{Cartesian Coordinates (Standard Orientation)}

$\begin{array}{lrrr}17 & 1.613453 & 0.096584 & 0.000000 \\ 16 & -0.425964 & -0.164636 & 0.000000 \\ 7 & -1.023630 & 1.338570 & -0.000004 \\ 8 & -0.736581 & -0.755053 & -1.248372 \\ 8 & -0.736582 & -0.755046 & 1.248375 \\ 1 & -0.831284 & 1.851532 & -0.848358 \\ 1 & -0.831290 & 1.851534 & 0.848350\end{array}$

Sulfamoyl fluoride $\left(\mathrm{NH}_{2} \mathrm{SO}_{2} \mathrm{~F}\right)$ at mPW1PW91/6311++G(3df,2p)

Energy: -704.506438080 Hartrees

Cartesian Coordinates (Standard Orientation)

$\begin{array}{lrrr}9 & 0.581473 & -0.000002 & 1.351393 \\ 16 & 0.085604 & 0.000001 & -0.146270\end{array}$




$\begin{array}{rrrr}7 & -1.513533 & -0.000004 & 0.015840 \\ 8 & 0.487060 & 1.247001 & -0.669659 \\ 8 & 0.487068 & -1.246995 & -0.669666 \\ 1 & -1.900608 & 0.852399 & 0.390753 \\ 1 & -1.900604 & -0.852410 & 0.390753\end{array}$

\section{Sulfonamide $\left(\mathrm{NH}_{2} \mathrm{SO}_{2} \mathrm{H}\right)$ at $\mathrm{mPW}$ PIPW91/6311++G(3df,2p)}

Energy: -605.230912768 Hartrees

\begin{tabular}{lrrr}
\multicolumn{4}{c}{ Cartesian Coordinates (Standard Orientation) } \\
1 & 1.340517 & 0.801752 & 0.000000 \\
16 & 0.253937 & -0.003391 & 0.000000 \\
7 & -1.012638 & 1.007707 & 0.000000 \\
8 & 0.253937 & -0.675711 & 1.254209 \\
8 & 0.253937 & -0.675711 & -1.254209 \\
1 & -1.189014 & 1.504963 & -0.857722 \\
1 & -1.189014 & 1.504963 & 0.857722
\end{tabular}

Sulfamic acid, neutral $\left(\mathrm{NH}_{2} \mathrm{SO}_{2} \mathrm{OH}\right)$ at mPW1PW91/6311++G(3df,2p)

Energy: -680.485089165 Hartrees

Cartesian Coordinates (Standard Orientation)

$\begin{array}{lrrr}8 & 0.724644 & 1.307258 & -0.006531 \\ 16 & 0.056978 & -0.148950 & 0.000678 \\ 7 & -1.509377 & 0.237770 & -0.000740 \\ 8 & 0.363515 & -0.775865 & -1.235430 \\ 8 & 0.364244 & -0.763844 & 1.242636 \\ 1 & -1.824957 & 0.678622 & -0.851267 \\ 1 & -1.824457 & 0.686717 & 0.845726 \\ 1 & 1.684184 & 1.213091 & -0.005531\end{array}$

Methane sulfonamide $\left(\mathrm{NH}_{2} \mathrm{SO}_{2} \mathrm{CH}_{3}\right)$ at $m P W 1 P W 91 / 6311++G(3 d f, 2 p)$

Energy: -644.562098017 Hartrees

Cartesian Coordinates (Standard Orientation)
$\begin{array}{lrrr}6 & -1.512611 & 0.664756 & -0.000169 \\ 16 & 0.051214 & -0.169933 & 0.000038 \\ 7 & 1.208593 & 0.982485 & -0.000248 \\ 8 & 0.166228 & -0.849577 & 1.250107 \\ 8 & 0.166229 & -0.850245 & -1.249663 \\ 1 & 1.303507 & 1.508827 & 0.853129 \\ 1 & 1.303583 & 1.508383 & -0.853890 \\ 1 & -2.277840 & -0.108600 & 0.000070 \\ 1 & -1.596422 & 1.271208 & -0.898335 \\ 1 & -1.596382 & 1.271761 & 0.897624\end{array}$

Phosphamyl bromide anion $\left(\mathrm{NH}_{2} \mathrm{PO}_{2} \mathrm{Br}^{-}\right)$at $m P W 1 P W 91 / 6311++G(3 d f, 2 p)$

Energy: -3122.32657835 Hartrees 


$\begin{array}{lrrr}\text { Cartesian Coordinates (Standard Orientation) } \\ 35 & 1.383778 & 0.008886 & 0.000000 \\ 15 & -1.012847 & -0.168424 & 0.000000 \\ 7 & -1.417409 & 1.463826 & -0.000001 \\ 8 & -1.321811 & -0.743747 & -1.318428 \\ 8 & -1.321811 & -0.743746 & 1.318428 \\ 1 & -1.084336 & 1.934260 & -0.828216 \\ 1 & -1.084337 & 1.934260 & 0.828214\end{array}$

Phosphamyl chloride anion $\left(\mathrm{NH}_{2} \mathrm{PO}_{2} \mathrm{Cl}\right)$ at $m P W 1 P W 91 / 6311++G(3 d f, 2 p)$

Energy: -1008.24949632 Hartrees

\begin{tabular}{lrrr}
\multicolumn{4}{c}{ Cartesian Coordinates (Standard Orientation) } \\
17 & 1.678749 & 0.038352 & 0.000000 \\
15 & -0.500838 & -0.172611 & 0.000000 \\
7 & -0.959910 & 1.449191 & -0.000002 \\
8 & -0.813984 & -0.754274 & -1.316693 \\
8 & -0.813984 & -0.754271 & 1.316695 \\
1 & -0.641524 & 1.930609 & -0.827762 \\
1 & -0.641525 & 1.930610 & 0.827759
\end{tabular}

Phosphamyl fluoride anion $\left(\mathrm{NH}_{2} \mathrm{PO}_{2} \mathrm{~F}\right)$ at $\mathrm{mPW}$ P PW91/6311++G(3df,2p)

Energy: -647.874957652 Hartrees

$\begin{array}{lrrr}\text { Cartesian Coordinates (Standard Orientation) } \\ 9 & -0.347653 & 0.000000 & 1.470803 \\ 15 & -0.130263 & 0.000000 & -0.144425 \\ 7 & 1.565217 & 0.000000 & -0.225168 \\ 8 & -0.613177 & -1.313698 & -0.616776 \\ 8 & -0.613176 & 1.313698 & -0.616776 \\ 1 & 1.968563 & -0.828161 & 0.186873 \\ 1 & 1.968563 & 0.828161 & 0.186873\end{array}$

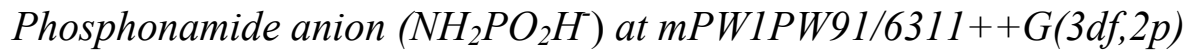

Energy: -548.553992616 Hartrees

$\begin{array}{lrrr}\text { Cartesian Coordinates (Standard Orientation) } \\ 1 & -0.148522 & 0.000000 & 1.637572 \\ 15 & -0.176639 & 0.000000 & 0.203213 \\ 7 & 1.488673 & 0.000000 & -0.206694 \\ 8 & -0.722460 & -1.319435 & -0.216265 \\ 8 & -0.722459 & 1.319435 & -0.216265 \\ 1 & 1.968375 & -0.829897 & 0.110668 \\ 1 & 1.968376 & 0.829896 & 0.110667\end{array}$

Hydrogen phosphamate anion $\left(\mathrm{NH}_{2} \mathrm{PO}_{2} \mathrm{OH}\right)$ at $\mathrm{mPW}$ P PW91/6311++G(3df,2p)

Energy: -623.834418516 Hartrees 


Cartesian Coordinates (Standard Orientation)
$\begin{array}{lrrr}8 & 0.556333 & 1.396692 & -0.354236 \\ 15 & 0.086721 & -0.157481 & 0.039818 \\ 7 & -1.599098 & 0.047829 & -0.011380 \\ 8 & 0.484332 & -1.045490 & -1.082083 \\ 8 & 0.485565 & -0.403832 & 1.448916 \\ 1 & -1.915814 & 0.316480 & -0.931562 \\ 1 & -1.914975 & 0.722551 & 0.669923 \\ 1 & 1.513828 & 1.409430 & -0.356742\end{array}$

Methyl phosphonamate anion $\left(\mathrm{NH}_{2} \mathrm{PO}_{2} \mathrm{CH}_{3}^{-}\right)$at $\mathrm{mPW}$ P PW91/6311++G(3df,2p)

Energy: -587.882530310 Hartrees

Cartesian Coordinates (Standard Orientation)
$\begin{array}{lrrr}6 & -1.574643 & 0.627904 & -0.000001 \\ 15 & 0.071238 & -0.199367 & 0.000003 \\ 7 & 1.200552 & 1.094848 & -0.000026 \\ 8 & 0.213416 & -0.882213 & 1.317348 \\ 8 & 0.213396 & -0.882262 & -1.317320 \\ 1 & 1.156919 & 1.666387 & 0.830565 \\ 1 & 1.156902 & 1.666366 & -0.830631 \\ 1 & -2.344068 & -0.145669 & -0.000026 \\ 1 & -1.704407 & 1.243942 & -0.891776 \\ 1 & -1.704427 & 1.243910 & 0.891793\end{array}$

Methanesulfonyl bromide $\left(\mathrm{CH}_{3} \mathrm{SO}_{2} \mathrm{Br}\right)$ at mPW1PW91/6311++G(3df,2p)

Energy: -3162.90722480 Hartrees

\begin{tabular}{lrrr}
\multicolumn{4}{l}{ Cartesian Coordinates (Standard Orientation) } \\
35 & 1.339365 & -0.047100 & 0.000000 \\
16 & -0.896419 & -0.137162 & 0.000000 \\
6 & -1.290640 & 1.585617 & 0.000000 \\
8 & -1.290640 & -0.710487 & -1.242423 \\
8 & -1.290640 & -0.710487 & 1.242423 \\
1 & -2.378921 & 1.632328 & 0.000000 \\
1 & -0.881041 & 2.032436 & 0.900216 \\
1 & -0.881041 & 2.032436 & -0.900216
\end{tabular}

Methanesulfonyl chloride $\left(\mathrm{CH}_{3} \mathrm{SO}_{2} \mathrm{Cl}\right)$ at mPW1PW91/6311++G(3df,2p)

Energy: -1048.83155281 Hartrees

\begin{tabular}{lrrr}
\multicolumn{4}{l}{ Cartesian Coordinates (Standard Orientation) } \\
17 & -1.622154 & -0.060886 & 0.000000 \\
16 & 0.428548 & -0.151067 & 0.000000 \\
6 & 0.818054 & 1.569168 & 0.000000 \\
8 & 0.818054 & -0.726029 & 1.240801 \\
8 & 0.818054 & -0.726029 & -1.240801 \\
1 & 1.905630 & 1.620055 & 0.000000 \\
1 & 0.408512 & 2.016774 & -0.899939
\end{tabular}


Methanesulfonyl fluoride $\left(\mathrm{CH}_{3} \mathrm{SO}_{2} \mathrm{~F}\right)$ at mPW1PW91/6311++G(3df,2p)

Energy: -688.458297067 Hartrees

Cartesian Coordinates (Standard Orientation)

$\begin{array}{llll}9 & -0.415994 & 1.416437 & -0.000009\end{array}$

$\begin{array}{llll}16 & -0.131411 & -0.133485 & 0.000001\end{array}$

$\begin{array}{llll}6 & 1.621394 & -0.067095 & -0.000001\end{array}$

$\begin{array}{llll}8 & -0.609420 & -0.625226 & 1.238543\end{array}$

$\begin{array}{llll}8 & -0.609423 & -0.625242 & -1.238534\end{array}$

$\begin{array}{lllr}1 & 1.968386 & -1.097532 & 0.000011\end{array}$

$\begin{array}{llll}1 & 1.950255 & 0.445830 & -0.898760\end{array}$

$\begin{array}{llll}1 & 1.950256 & 0.445851 & 0.898746\end{array}$

S-H-sulfinic acid $\left(\mathrm{CH}_{3} \mathrm{SO}_{2} \mathrm{H}\right)$ at mPW1PW91/6311++G(3df,2p)

Energy: -589.178074634 Hartrees

\begin{tabular}{lrrr}
\multicolumn{4}{c}{ Cartesian Coordinates (Standard Orientation) } \\
1 & -1.355822 & 0.807397 & 0.000000 \\
16 & -0.275471 & -0.018996 & 0.000000 \\
6 & 1.042227 & 1.153887 & 0.000000 \\
8 & -0.275471 & -0.720064 & 1.247869 \\
8 & -0.275471 & -0.720064 & -1.247869 \\
1 & 1.964218 & 0.575474 & 0.000000 \\
1 & 0.976651 & 1.759382 & -0.900419 \\
1 & 0.976651 & 1.759382 & 0.900419
\end{tabular}

Methanesulfonic acid $\left(\mathrm{CH}_{3} \mathrm{SO}_{2} \mathrm{OH}\right)$ at mPW1PW91/6311++G(3df,2p)

Energy: -664.436497573 Hartrees

Cartesian Coordinates (Standard Orientation)
$\begin{array}{lrrr}8 & 0.546001 & 1.397711 & -0.000036 \\ 16 & 0.104678 & -0.138302 & 0.000002 \\ 6 & -1.630213 & 0.133461 & 0.000007 \\ 8 & 0.507533 & -0.732946 & -1.231451 \\ 8 & 0.507552 & -0.732888 & 1.231477 \\ 1 & -2.088602 & -0.852179 & 0.000023 \\ 1 & -1.901363 & 0.679627 & 0.898170 \\ 1 & -1.901372 & 0.679602 & -0.898168 \\ 1 & 1.509069 & 1.450003 & -0.000015\end{array}$

Methylphosphonyl bromide anion $\left(\mathrm{CH}_{3} \mathrm{PO}_{2} \mathrm{Br}^{-}\right)$at mPW1PW91/6311++G(3df,2p)

Energy: -3106.26262319 Hartrees

Cartesian Coordinates (Standard Orientation)

$\begin{array}{rrrr}35 & -1.388753 & -0.011553 & 0.000000 \\ 15 & 0.992745 & -0.182174 & 0.000000\end{array}$




$\begin{array}{rrrr}6 & 1.338454 & 1.600965 & 0.000000 \\ 8 & 1.338454 & -0.769539 & 1.309822 \\ 8 & 1.338454 & -0.769539 & -1.309822 \\ 1 & 2.423524 & 1.719587 & 0.000000 \\ 1 & 0.922817 & 2.062108 & -0.892661 \\ 1 & 0.922817 & 2.062108 & 0.892661\end{array}$

Methylphosphonyl chloride $\left(\mathrm{CH}_{3} \mathrm{PO}_{2} \mathrm{Cl}\right)$ at mPW1PW91/6311++G(3df,2p)

Energy: -992.186098244 Hartrees

\begin{tabular}{lrrr}
\multicolumn{4}{l}{ Cartesian Coordinates (Standard Orientation) } \\
17 & -1.686326 & -0.025525 & 0.000000 \\
15 & 0.484988 & -0.181597 & 0.000000 \\
6 & 0.845235 & 1.599852 & 0.000000 \\
8 & 0.845235 & -0.767920 & 1.308090 \\
8 & 0.845235 & -0.767920 & -1.308090 \\
1 & 1.930377 & 1.712798 & 0.000000 \\
1 & 0.433588 & 2.066345 & -0.892417 \\
1 & 0.433588 & 2.066345 & 0.892417
\end{tabular}

Methylphosphonyl fluoride $\left(\mathrm{CH}_{3} \mathrm{PO}_{2} \mathrm{~F}^{-}\right)$at mPW1PW91/6311++G(3df,2p)

Energy: -631.812714372 Hartrees

\begin{tabular}{lrrr}
\multicolumn{4}{l}{ Cartesian Coordinates (Standard Orientation) } \\
9 & -0.561860 & 1.415626 & 0.000000 \\
15 & -0.137337 & -0.153438 & 0.000000 \\
6 & 1.667259 & 0.091992 & 0.000000 \\
8 & -0.561860 & -0.711052 & 1.305071 \\
8 & -0.561860 & -0.711052 & -1.305071 \\
1 & 2.146627 & -0.886611 & 0.000000 \\
1 & 1.978186 & 0.636207 & -0.891409 \\
1 & 1.978186 & 0.636207 & 0.891409
\end{tabular}

Methylphosphinate aniona $\left(\mathrm{CH}_{3} \mathrm{PO}_{2} \mathrm{H}\right)$ at mPW1PW91/6311++G(3df,2p)

Energy: -532.488721165 Hartrees

Cartesian Coordinates (Standard Orientation)

$\begin{array}{lrrr}1 & -1.410061 & 0.890410 & 0.000000 \\ 15 & -0.298534 & -0.023713 & 0.000000 \\ 6 & 1.088986 & 1.179289 & 0.000000 \\ 8 & -0.298534 & -0.739878 & 1.311868 \\ 8 & -0.298534 & -0.739878 & -1.311868 \\ 1 & 2.025313 & 0.618414 & 0.000000 \\ 1 & 1.052700 & 1.804590 & -0.893619 \\ 1 & 1.052700 & 1.804590 & 0.893619\end{array}$

Hydrogen methylphosphonate anion $\left(\mathrm{CH}_{3} \mathrm{PO}_{2} \mathrm{OH}\right)$ at mPW1PW91/6311++G(3df,2p)

Energy: -607.771821334 Hartrees 


\begin{tabular}{lrrr}
\multicolumn{4}{l}{ Cartesian Coordinates (Standard Orientation) } \\
8 & 0.559753 & 1.453111 & -0.000058 \\
15 & 0.117683 & -0.150708 & 0.000004 \\
6 & -1.683950 & 0.124105 & 0.000005 \\
8 & 0.530719 & -0.755433 & -1.297718 \\
8 & 0.530738 & -0.755332 & 1.297767 \\
1 & -2.171727 & -0.850399 & 0.000045 \\
1 & -1.988431 & 0.671786 & 0.891427 \\
1 & -1.988442 & 0.671719 & -0.891454 \\
1 & 1.517380 & 1.484119 & -0.000041
\end{tabular}




\section{References for Supporting Information}

(1) Pangborn, A. B.; Giardello, M. A.; Grubbs, R. H.; Rosen, R. K.; Timmers, F. J. Organometallics 1996, 15, 1518-1520.

(2) Yamada, K.; Kurokawa, T.; Tokuyama, H.; Fukuyama, T. J. Am. Chem. Soc. 2003, 125, 6630-6631.

(3) Looker, J. H. J. Org. Chem. 1952, 17, 510-514.

(4) Suh, J. H.; Suh, M. K.; Cho, S. H. J. Chem. Soc., Perkin Trans. 2 1990, $685-688$.

(5) Percec, V.; Bae, J. Y.; Zhao, M. Y.; Hill, D. H. J. Org. Chem. 1995, 60, 176-185.

(6) Kaboudin, B. Phosphorus, Sulfur Silicon Relat. Elem. 2003, 178, 887-892.

(7) Carnahan, J. C.; Closson, W. D.; Ganson, J. R.; Juckett, D. A.; Quaal, K. S. J. Am. Chem. Soc. 1976, 98, 2526-2531.

(8) Allen, J. J.; Lazerwith, S. E.; Shokat, K. M. J. Am. Chem. Soc. 2005, 127, 5288-5289.

(9) Dietze, P.; Jencks, W. P. J. Am. Chem. Soc. 1989, 111, 5880-5886.

(10) CRC Handbook of Chemistry and Physics; 86 ed.; CRC Press: Boca Raton, 2005.

(11) Nakagawa, Y.; Uehara, K.; Mizuno, N. Inorg. Chem. 2005, 44, 90689075. 